\title{
Tu parles! Et puis quoi encore! Phrases préfabriquées à fonction expressive dans les dictionnaires français
}

\author{
Agnès TUTIN* \\ LInguistique et DIdactique des Langues Étrangères et Maternelles, Université Grenoble Alpes - \\ EA609
}

\begin{abstract}
Résumé. Les formules expressives, par exemple tu parles! et puis quoi encore!, constituent un sous-ensemble de phrases préfabriquées pour lesquelles la fonction expressive et émotionnelle apparaît essentielle. Cette étude analyse comment ce type d'expression est modélisé dans des dictionnaires de langue de référence et des dictionnaires phraséologiques. Les résultats montrent que, malgré des incohérences et des lacunes, certains ouvrages lexicographiques recèlent des informations précieuses pour l'étude de ces phénomènes phraséologiques.
\end{abstract}

\begin{abstract}
You bet! What's next? Prefabricated sentences with expressive function in French dictionaries. Expressive formulas are a subset of prefabricated sentences for which the expressive and emotional function appears essential. This study analyses how this type of expression is described in reference language dictionaries and phraseological dictionaries. The results show that, despite inconsistencies and gaps, some dictionaries contain valuable information for the study of these phraseological phenomena.
\end{abstract}

\section{Introduction}

Depuis quelques années, le domaine de la phraséologie se renouvelle et étend à la fois ses objets linguistiques (des mots composés aux constructions et aux phrases) et ses méthodes d'analyse. Dans ce cadre, la phraséologie pragmatique, qui traite des phrases préfabriquées associées à des contraintes interactionnelles, a le vent en poupe.

Dans cet article, nous nous intéressons à un type particulier de phraséologismes pragmatiques, les phrases à fonction expressive ou formules expressives, qui ont été l'objet d'un projet de recherche franco-polonais appelé PRAGMALEX. L'objectif de ce projet était de proposer une réflexion sur ce type de phénomène et de mettre au point une description d'un sous-ensemble représentatif de ces expressions en français, italien et polonais

*agnes.tutin@univ-grenoble-alpes.fr 
(Krzyżanowska \& Grossmann, 2019 et à paraître). Les phraséologismes pragmatiques qui nous intéressent ont pour principale fonction de véhiculer une émotion ou d'exprimer un point de vue de la part du locuteur (cf. aussi Kauffer, 2013 ; Dostie, 2019 ; Tutin, 2019). Il s'agit de phrases toutes faites comme : C'est une honte! Et puis quoi encore. Ça va pas la tête!

L'objectif est ici d'observer dans quelle mesure et comment ces expressions sont traitées dans les dictionnaires de langue classiques du français, mais aussi de quelques dictionnaires phraséologiques de référence. Cette étude a un double objectif : d'une part, elle cherche à repérer si une certaine cohérence peut être mise en évidence pour ces objets phraséologiques encore mal circonscrits; d'autre part, la comparaison des dictionnaires pourra permettre d'orienter le linguiste vers les dictionnaires les plus adaptés pour l'étude des phrases expressives.

Dans un premier temps, nous reviendrons sur les contours des « formules expressives » dans le «maquis» des phraséologismes pragmatiques. Nous présenterons ensuite la liste d'expressions choisies, le corpus de dictionnaires et les paramètres qui seront examinés. Nous analyserons ensuite les dictionnaires et proposerons une synthèse de nos observations.

\section{Les phrases préfabriquées à fonction expressive dans le maquis des phraséologismes pragmatiques}

Les phénomènes qui nous intéressent ne sont pas nouveaux dans le domaine de la phraséologie. Ils étaient déjà décrits sous le terme de "locution exclamative » par Bally (1909), même s'ils n'ont pas fait l'objet de descriptions fines dans sa typologie des phraséologismes. La phraséologie pragmatique fait l'objet d'un nombre croissant d'études dans le champ francophone depuis quelques années, ce qui s'accompagne d'un foisonnement terminologique : "énoncés liés 》 de Fónagy (1995), "énoncés de motifs usuels 》 (MartinsBaltar, 1995), « routines conversationnelles » (Klein \& Lamiroy, 2011), « actes de langage stéréotypés » (Kauffer, 2013 ; 2019), «pragmatèmes » (Mel’čuk, 2013; Blanco \& Mejri, 2018).

Ces phraséologismes pragmatiques présentent plusieurs caractéristiques :

- ce sont des énoncés complets,

- dont la réalisation lexicale est peu prédictible, mais liée à une situation de production spécifique,

- ayant une fonction « actionnelle » (acte expressif, directif, promissif...), particulièrement présents à l'oral et

- ayant parfois des caractéristiques gestuelles ${ }^{1}$.

Prenons quelques exemples pour illustrer plus concrètement cette définition.

(1) Qu'est-ce que ce sera?

est la formulation stéréotypée d'un commerçant qui s'adresse à un client pour lui demander l'achat qu'il souhaite effectuer.

(2) Ça alors!

exprime la surprise du locuteur suite à la prise de conscience d'un fait.

\section{(3) Comment dirais-je}

est une formule métalinguistique fréquente dans la conversation lorsque le locuteur « cherche ses mots ». 
On notera que ces phrases usuelles présentent des contraintes linguistiques dans leur réalisation dans la mesure où le locuteur n'est pas libre de choisir n'importe quel lexème pour réaliser l'énoncé. Le commerçant pourra employer d'autres formulations que (1) comme qu'est-ce que vous voudrez? mais elles apparaîtront moins naturelles, voire incongrues ${ }^{2}$. De la même façon, celui qui cherche une formulation adéquate pourra bien entendu dire : je ne sais pas comment dire ça, mais la phrase paraîtra plus maladroite et moins idiomatique que comment dire ou comment dirais-je.

Parmi ces phrases préfabriquées, nous nous intéressons ici particulièrement aux phrases de type (2), dans lesquelles la fonction expressive et émotionnelle apparaît prépondérante comme il manquerait plus que ça! ça va pas la tête! c'est le pied. Ces formules sont bien entendu particulièrement productives dans les interactions orales et relèvent souvent d'un registre familier, mais contrairement à d'autres phrases préfabriquées comme y a pas de quoi ou c'est clair!, elles peuvent apparaître dans un contexte monologal, en réaction à un événement ou à un fait (Dostie 2019).

Par comparaison aux formules expressives, les phrases comme (1) peuvent être considérées comme des pragmatèmes au sens de Mel'čuk et Blanco (par exemple, Mel'čuk 2013; Blanco \& Mejri, 2018), car elles sont extrêmement contraintes par la situation de production (ici, chez un commerçant qui s'adresse à un client). Dostie (2019) utilise pour ce type d'expression le terme de " phrase sociale » qui nous paraît particulièrement approprié, car la dimension culturelle et interactionnelle apparaît ici essentielle. Les phrases de type (3) ont davantage une fonction métadiscursive ou métaénonciative. Une de leurs caractéristiques est de souvent apparaître comme phrase parenthétique et non comme énoncés autonomes ${ }^{3}$.

\section{Corpus d'étude et objectifs}

\subsection{Objectifs}

Cette recherche dans les dictionnaires poursuit plusieurs objectifs. La première est tout d'abord de déterminer dans quelle mesure les formules qui nous intéressent sont répertoriées dans ces ouvrages. Peut-on faire l'hypothèse, à la suite de l'étude de Blanco \& Mejri (2018) sur les pragmatèmes qu'elles seront peu traitées, car elles relèvent plutôt du code oral et qu'elles sont partiellement compositionnelles ? On cherchera aussi à observer si le caractère préfabriqué de ces expressions est établi. Il sera également pertinent de repérer si le traitement des formules présente une certaine cohérence au sein des ouvrages. Enfin, on pourra observer le type de traitement pragmatique et sémantique proposé, quand il est présent.

\subsection{Choix des phrases préfabriquées}

Pour cette étude exploratoire, un corpus de 10 phrases préfabriquées a été sélectionné 4 . Ces expressions font pour la plupart partie de la liste des 50 phrases sélectionnées dans le cadre du projet PRAGMALEX ${ }^{5}$. Ces phrases sont assez courantes et productives dans les corpus oraux, comme le corpus Corpus d'Étude pour le Français Contemporain (CEFC) du projet $\mathrm{ORFEO}^{6}$ (Debaisieux et al., 2016).

\footnotetext{
- $\quad$ C'est charmant

- C'est malin

- C'est pas croyable

- C'est pas possible
} 
$\begin{array}{ll}\text { - } & \text { C'est pas vrai } \\ \text { - } & \text { C'est une honte } \\ \text { - } & \text { C'est une plaie } \\ \text { - } & \text { Encore heureux } \\ \text { - } & \text { Tu parles } \\ \text { - } & \text { Tu peux toujours courir }\end{array}$

Ces formules ont pour la plupart été analysées dans le cadre du projet PRAGMALEX, ce qui nous permet de disposer d'une description de référence pour l'étude des dictionnaires (cf. définitions des formules en annexe). On peut observer que les formules retenues correspondent à des patrons syntaxiques récurrents (en dehors de encore heureux), qui sont fréquents pour ces expressions : c'est Adj; c'est pas Adj; c'est un $\mathrm{N} ; T u \mathrm{~V}$. Bien entendu, ces expressions peuvent aussi se rencontrer dans un sens non formulaire dans les dictionnaires, comme dans l'exemple 1 extrait du Petit Robert.

Exemple 1. Un exemple avec c'est charmant au sens littéral dans le PR (s.v. charmant)

Dans cette étude, c'est exclusivement l'emploi formulaire expressif qui nous intéressera, par exemple l'emploi ironique de c'est charmant ou c'est malin, comme celui qui apparaît dans l'exemple 2, extrait du Petit Robert.

- PAR ANTIPHR. C'est malin! Tu peux être fier de toi ! c'est stupide.

Exemple 2. Le traitement de c'est malin au sens formulaire dans le PR (s.v. malin)

\subsection{Les dictionnaires}

Deux types de dictionnaires ont été choisis. D’une part, nous avons sélectionné des dictionnaires de langue qui constituent des références pour le français :

a) Le Grand Robert, dictionnaire de référence contemporain de langue en 6 volumes. La version électronique a été utilisée 7.

b) Le Petit Robert en ligne (édition 2019, désormais PR), qui est une version abrégée du Grand Robert.

c) Le Trésor de la Langue Française (version informatique, désormais TLFi), grand dictionnaire de référence du français en 16 volumes, achevé en 1994, et librement accessible en ligne ${ }^{8}$.

d) Nous avons ajouté à ces ouvrages classiques le dictionnaire collaboratif Wiktionnaire $^{9}$, qui est un dictionnaire exclusivement électronique librement accessible sur la toile et dont les articles sont construits de façon collaborative.

D'autre part, nous avons également intégré dans notre étude des dictionnaires plus spécialisés de phraséologie. Parmi ceux-ci, nous avons sélectionné trois ouvrages :

a) le Dictionnaire d'expressions et locutions, Alain Rey et Sophie Chantreau, Le Robert (2003, désormais DEL), qui constitue une référence dans le domaine de la phraséologie, et

b) le Dictionnaire du français parlé : le monde des expressions familières, Charles Bernet \& Pierre Rézeau, Seuil (1989, désormais DFP), qui intègre de nombreuses expressions du français parlé. 
c) Enfin, un ouvrage à coloration plus universitaire a été intégré à notre étude : Structures figées de la conversation, Françoise Bidaud, Peter Lang (2002, désormais SFC). Ce dernier ouvrage recense plus de 2000 formules de la conversation, qui sont décrites de façon assez fine et présentent des équivalents italiens et des exemples d'emplois.

Dans ces trois ouvrages spécialisés, les expressions sont rangées à partir d'un mot clé de l'expression qui sert de vedette. Par exemple, l'expression c'est une plaie dans le SFC apparaît sous le lexème PLAIE. L'article comporte une étiquette sémantique (E.S.), des équivalents en italien (E.P.), un commentaire sur l'usage et un exemple (fabriqué) d'emploi, parfois des citations.

PLAIE. - C'est une [vraie] plaie!

[E.S. Plainte - E.P. E' une calamità !/E' una piagia !]

Commentaire qui s'applique à une situation désastreuse, à une " chose très pénible, qui a des conséquences très graves" (le Robert) ou, familièrement, à un personnage particulièrement insupportable.

- - Il est sympathique? - Ce garcon? Personne ne le supporte, c'est une vraie plaie!

Exemple 3. L'expression c'est une [vraie] plaie dans les Structures figées de la conversation (Bidaud 2002)

\section{Résultats et analyse}

\subsection{Les dictionnaires de langue}

\subsubsection{Mention de l'expression}

Dans un premier temps, nous avons simplement observé dans quelle mesure l'expression était mentionnée, quel que soit le champ (entrée à part entière, syntagme défini, syntagme non défini, exemple). Dans l'ensemble, les expressions sélectionnées apparaissent bien répertoriées puisque $82,5 \%$ d'entre elles $\left(33 / 40^{10}\right)$ sont mentionnées dans les dictionnaires. Ce pourcentage (qui serait à vérifier sur un corpus de plus grande ampleur) semble supérieur à la proportion signalée par Blanco \& Mejri (2018) pour les pragmatèmes, qui ne mentionnent que $30 \%$ de pragmatèmes répertoriés. On peut faire l'hypothèse (à confirmer sur un plus large panel de formules) que la mention est d'autant plus fréquente que l'expression est opaque. 


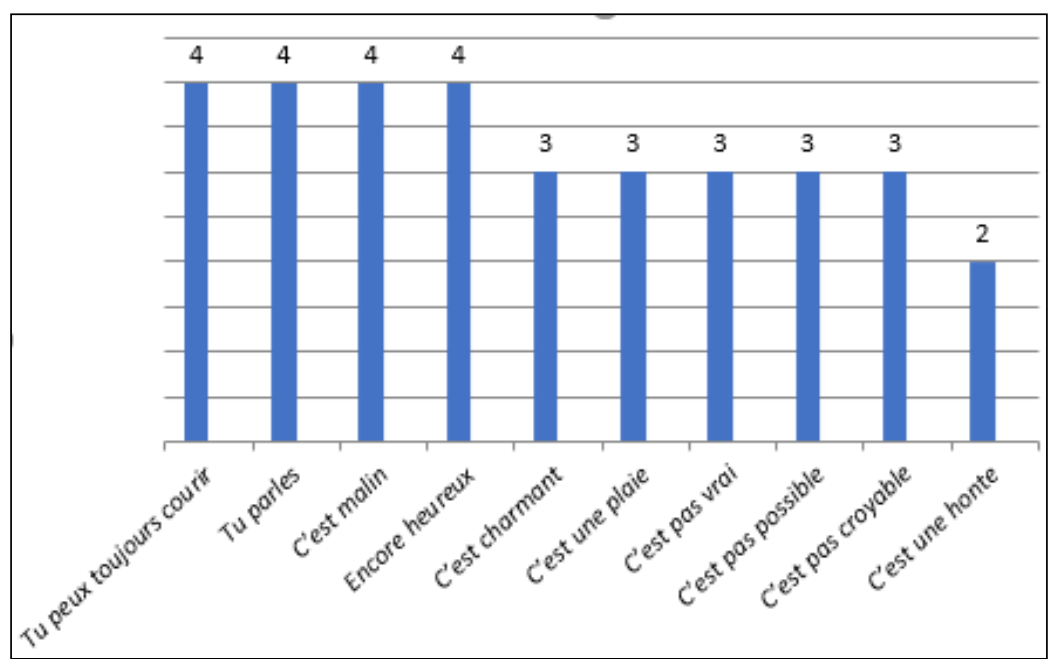

Figure 1. Mention des expressions dans les quatre dictionnaires de langue

Si l'on observe maintenant la répartition des mentions selon les dictionnaires, c'est contre toute attente les GR et le PR qui les recensent le mieux, même si le traitement y est, comme on le verra, parfois assez rudimentaire.

\begin{tabular}{|l|l|l|l|}
\hline GR & PR & TLF & Wiktionnaire \\
\hline 10 & 10 & 7 & 5 \\
\hline
\end{tabular}

Tableau 1. Répartition des 10 expressions dans les 4 dictionnaires de langue.

\subsubsection{Champ d'occurrence de l'expression}

Comme attendu, les formules expressives apparaissent dans plusieurs champs. Parmi nos trois dictionnaires, seul Wiktionnaire traite certaines de ces expressions comme des entrées à part entière ${ }^{11}$. L'exemple 4 présente ainsi l'article de tu parles dans le Wiktionnaire. Ce traitement n'est toutefois pas systématique, car il ne concerne que 3 formules sur les 10 examinées. 


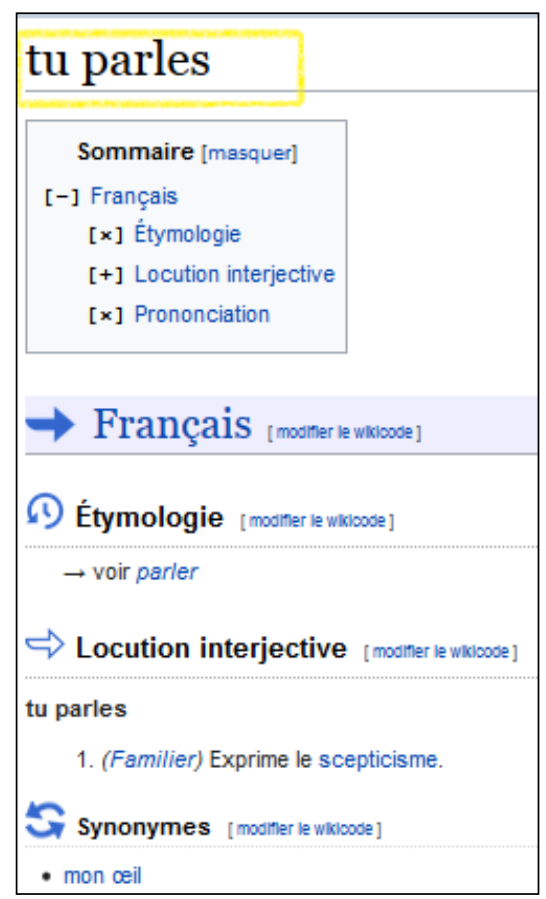

Exemple 4. L'article de tu parles dans le Wiktionnaire

Dans les autres cas, les formules apparaissent dans différents champs liés au mot simple de l'expression qui sert de vedette. Par exemple, ce n'est pas possible sera indiqué s.v. possible et encore heureux s.v. heureux. Au sein de l'article, les formules peuvent être mentionnées comme des exemples d'acceptions (comme ce n'est pas possible dans l'exemple 5), principalement dans le PR et le Wiktionnaire (9 cas).

\section{- (Pour marquer l'étonnement). Est-ce possible ? Ce n'est pas possible ! $\rightarrow$ croyable. (1790) ELLIPT et FAM. « Simone ponctuant ses silences de: "Non? - Pas possible ! - Pas vrai !" qui coupaient votre}

Exemple 5. La formule sous forme d'exemple d'acception (ex de ce n'est pas possible dans le PR)

La formule peut apparaître aussi comme un syntagme non défini dans le TLFi (Hausmann, 1996). L'expression est dans ce cas isolée dans un champ spécifique (ce n'est pas un exemple), mais elle ne reçoit pas de glose ou de définition spécifique (2 cas de ce type). C'est ainsi le cas de la formule encore heureux (s.v. heureux) dans l'exemple 6, qui reçoit par ailleurs des indications détaillées sur la construction syntaxique.

1. Pop. et fam. [P. ell. du verbe être] Encore heureux de (+ subst. ou inf.)/que (+ prop.). Pour le tirage au sort, j'ai amené un treize... Encore heureux que j'ai pas parti... (MÉTÉNIER, Lutte pour amour, 1891, p. 30). Ils ont pour moi tous les mépris. Encore heureux de pas être viré (cḱLns, Mort à crédit, 1936, p. 18)

Exemple 6. La formule encore heureux comme syntagme non défini dans le TLFi.

Enfin, de nombreuses formules sont des syntagmes définis. Ils sont ainsi suivis d'une glose ou d'une définition. Ce traitement apparaît dans le PR, le GR et le TLFi (13 cas), L'exemple 7 présente l'expression c'est une honte accompagnée d'une définition. 


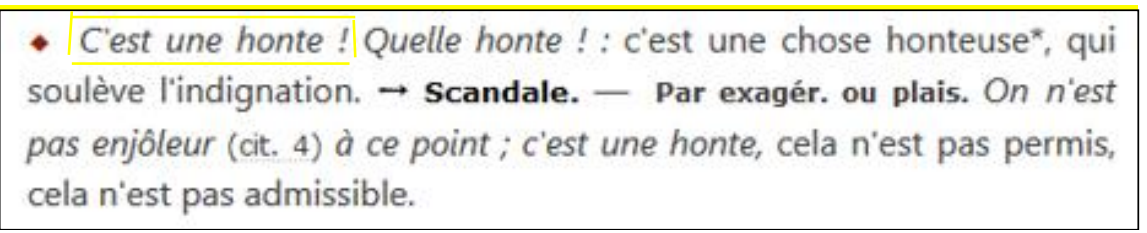

Exemple 7. La formule c'est une honte comme syntagme défini dans le GR (s.v. honte)

Au final, on observe une grande diversité, même au sein d'un même dictionnaire, pour la présentation des formules.

\subsubsection{Traitement pragmatique et sémantique}

Un traitement pragmatique et sémantique est proposé pour les formules qui apparaissent comme des entrées à part entière ou des syntagmes définis. Plusieurs articles comportent des indications d'emploi, qui renvoient à l'acte de parole et sont introduites par des verbes à fonction métalinguistique comme marquer, se dit, exprimer ... Le TLFi présente une certaine cohérence dans la présentation de ces éléments qui apparaissent toujours entre crochets, comme dans les exemples 8 et 9. L'indication d'emploi peut intégrer des informations sur le type d'émotion véhiculé par la formule (marque la surprise ... pour exprimer la stupeur ...) ou des informations sur le contexte interactionnel (En réplique dans un dialogue ...).

- Fam. Tu parles, vous parlez de, d'un(e)... [Marque la surprise l'irritation, l'admiration du locuteur] -(...) Dis donc, t'as entendu, c'te nuit, l'attaque? Mon vieux, tu parles d'un bombardement qu'ils ont balancé. Quelque chose de soigné comme décoction! (Barbusse, Feu, 1916, p.12). C'est malheureux de voir un tacot pareil! de Bayonne ici, quatre heures, quatre et deux font six. Six heures pour 180 kilomètres, vous parlez d'une moyenne! les gars du tour de France font mieux... (Bernanos, Crime, 1935, p.861).

Exemple 8. Indication d'emploi de la formule tu parles dans le TLFi (s.v. parler)

- Ce n'est pas vrai! ou, fam., c'est pas vrai! [En réplique dans un dialogue, pour exprimer la stupeur, la réprobation face à une nouvelle] Fanny: Je vais avoir un enfant. Le docteur vient de me le dire. Honorine: Ah! mon Dieu! Ah! mon Dieu! (...) Ce n'est pas possible! Ce n'est pas vrai! (...) Va-t'en, fille malhonnête! (pagnol, Fanny, 1932, I, $2^{\mathrm{e}}$ tabl., 6, p. 92). [Pour exprimer la surprise, l'étonnement] - (...) Quand elle est arrivée à Paris de sa cambrousse en Bretagne, elle a eu le béguin pour un petit gars, un émigré, un Polak, je crois. Il était gentil, ils avaient décidé de se marier. - C'est pas vrai! - Je te jure que si (Chr. aventn, Le Cour en poche, 1989, p. 165).

Exemple 9. Indications d'emploi de la formule c'est pas vrai dans le TLFi (s.v. vrai)

Dans le PR et le GR, l'indication d'emploi, moins fréquente, apparaît à travers un commentaire entre parenthèses, comme dans l'exemple 10.

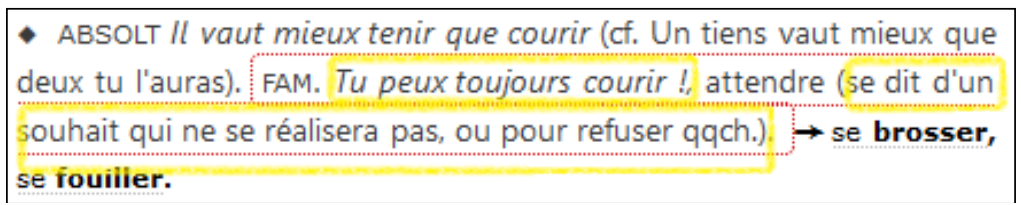

Exemple 10. Indication d'emploi de la formule tu peux toujours courir dans le PR (s.v. courir) 
On observe par ailleurs des gloses ou de courtes définitions qui paraphrasent la formule. La glose de l'exemple 11 pour le TLFi est assez atypique, avec une formulation paraphrastique empruntant la même structure que la formule.

c) Loc. [Avec ell. du compl. prép.]

- Fam. Tu peux toujours courir! Tu auras beau faire, tu n'obtiendras pas ce que tu veux.

Exemple 11. La glose de la formule tu peux toujours courir dans le TLFi (s.v. courir)

La mention de formules synonymes peut par ailleurs alterner avec des gloses plus neutres, comme dans l'exemple 12, extrait du PR.

\section{pas vrai ? n'est-ce pas ? FAM. Il est commerçant, pas vrai ? FAM. C'est pas vrai ! c'est pas possible ! sans blague ! ; c'est intolérable, excessif. Il est pas vrai, ce mec!}

Exemple 12. Gloses de la formule c'est pas vrai dans le PR (s.v. vrai)

$\mathrm{Au}$ terme de cet examen, on observe que les dictionnaires de langue présentent des informations assez riches sur les formules expressives, même si le codage manque de cohérence et de systématicité. Le TLFI se démarque avec des indications d'emploi plus détaillées et l'ajout de certaines informations syntaxiques. Qu'en est-il des dictionnaires phraséologiques?

\subsection{Les dictionnaires phraséologiques}

\subsubsection{Mention des formules expressives dans les ouvrages spécialisés}

De façon surprenante, les dictionnaires phraséologiques contiennent moins de mentions des formules expressives que les dictionnaires de langue. On n'y dénombre que 16 formules sur les 30 (10 expressions pour 3 dictionnaires), soit 53\% des occurrences (comme on le voit dans la Figure 2). Parmi ces ouvrages, sans surprise, c'est le SFC qui établit le meilleur inventaire des formules (Tableau 2). Le Dictionnaire du français parlé n'en répertorie que peu.

\begin{tabular}{|l|l|l|}
\hline SFC & DFP & DEL \\
\hline 8 & 3 & 5 \\
\hline
\end{tabular}

Tableau 2. Répartition des 10 expressions dans les 3 dictionnaires phraséologiques 


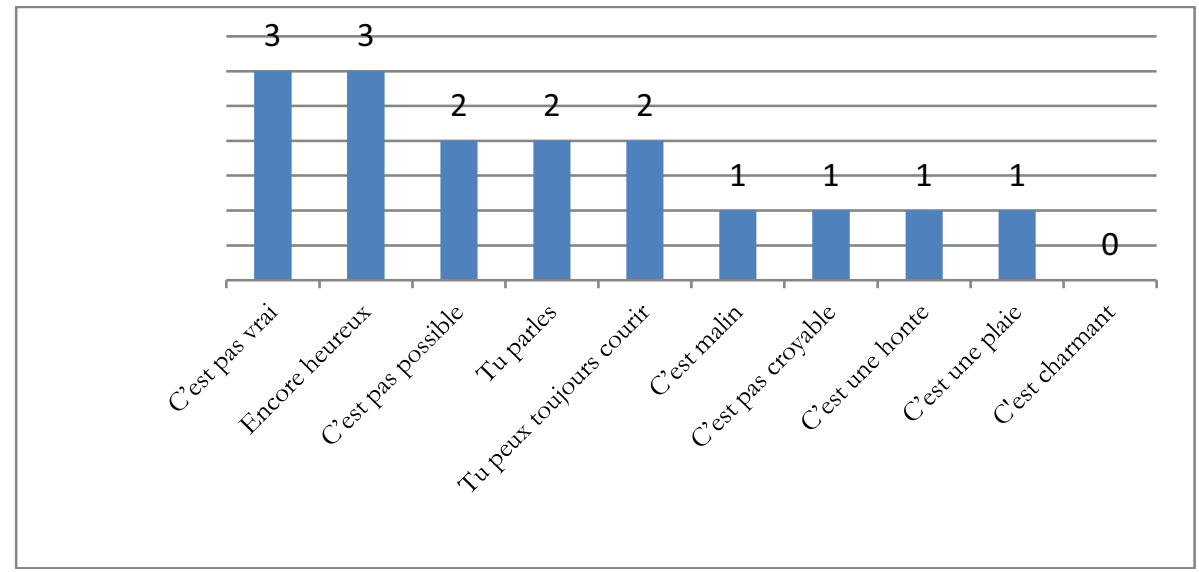

Figure 2. Mention des expressions dans les trois dictionnaires phraséologiques

\subsubsection{Type de champ et de traitement}

Dans les dictionnaires phraséologiques, les formules font la plupart l'objet d'entrée spécifique à part entière (sous la vedette d'un mot-clé) (14 cas sur 16), par exemple dans l'article du SFC de tu peux toujours courir!

189. COURIR - Tu peux toujours courir!

[E.S. Impossibilité / Refus - E.P. Stai fresco (se credo che ...)]

(pop.) L'expression peut se conjuguer au futur, et à toutes les personnes, elle sert à affirmer qu'un souhait ne pourra pas se réaliser ;

$\mid \ldots]$

Exemple 13. La formule tu peux toujours courir dans le SFC

Certaines d'entre elles sont mentionnées comme une variante ${ }^{12}$ (2 cas sur 16), comme c'est une honte.

\section{Honte}

o C'est la honte/Ça fait la honte/La honte ! Formule employée pour manifester un sentiment d'abaissement, d'humiliation, d'infériorité, de dégoût. Variante de c'est une honte, ça fait honte, quelle honte !

Exemple 14. La formule c'est une honte comme variante dans le DFP

\subsubsection{Traitement pragmatique et sémantique}

De façon attendue, le traitement pragmatique et sémantique est plus approfondi dans les dictionnaires phraséologiques que dans les dictionnaires de langue. D'une part, on observe des indications d'emploi assez détaillées, en particulier dans le SFC. Ces commentaires précisent les situations d'emploi et les actes de langage associés, en particulier les actes expressifs. Ces commentaires emploient la métalangue comme dans les exemples suivants : "Commentaire qui s'applique à une situation ... », «Formule employée pour manifester ...», «Formule par laquelle on manifeste ...», "Antiphrase qui sert de commentaire ironique ...», comme dans les exemples 15 et 16. 


\section{C'est pas Croyable !}

[E.S. Surprise / Indignation - E.P. E' incredibile !]

Exclamation devant une chose qui surprend ou révolte tellement que c'en est incroyable.

Souvent suivi de l'expansion ce que ...

$\lceil\ldots\rceil$

Exemple 15. Indication d'emploi de la formule c'est pas croyable dans le SFC

C'est pas vrai ! «ce n'est pas possible! sans blague !». S'emploie dans le code oral pour exprimer la surprise, l'étonnement, et par extension l'admiration ou la vive désapprobation, selon les contextes. La fréquence de cette expression depuis 1968-1970 est devenue très élevée $[\ldots]$

Exemple 16. Indication d'emploi de la formule c'est pas vrai dans le DEL

À côté des indications d'emploi, nombre d'articles, en particulier dans le SFC, comportent aussi des gloses qui paraphrasent l'expression, et proposent souvent un équivalent.

189. COURIR - Tu peux toujours courir !

[E.S. Impossibilité / Refus - E.P. Stai fresco (se credo che ...)]

(pop.) L'expression peut se conjuguer au futur, et à toutes les personnes, elle sert à affirmer qu'un souhait ne pourra pas se réaliser ; la glose est : tu as beau courir, tu n'obtiendras rien. Sens : « tes efforts ne serviront à rien. » [....]

Exemple 17. La glose de la formule tu peux toujours courir dans le SFC

Le SFC, spécialisé dans les formules de la conversation, présente donc une description sémantique et pragmatique assez complète - avec un métalangage simple destiné au grand public - des phénomènes qui nous intéressent.

\subsubsection{Informations grammaticales et autres}

Les dictionnaires spécialisés, de la même façon que le TLFi, intègrent souvent, et particulièrement le SFC, des remarques d'ordre grammatical. Ces éléments sont essentiels, car nombre de ces formules ne sont pas complètement figées : elles connaissent d'une part quelques variations d'ordre morphologique (il ne manquerait/manquait plus que ça), d'autre part, des expansions (tu parles de SN; c'est une honte de Vinf ....). Le SFC, et plus marginalement le DEL, comportent des indications de ce type, comme dans les exemples 18 et 19 .

189. COURIR - Tu peux toujours courir!

[E.S. Impossibilité / Refus - E.P. Stai fresco (se credo che ...)]

(pop.) L'expression peut se conjuguer au futur, et à toutes les personnes, elle sert à affirmer qu'un souhait ne pourra pas se réaliser ; $\lceil\ldots\rceil$

Exemple 18. Informations grammaticales sur tu peux toujours courir dans le SFC 


\section{Tu parles!}

[....] L'expression accepte une expansion introduite par la préposition de + substantif ou silcomme + syntagme verbal et prend alors souvent une forme exclamative. Tu parles d'un idiot $[\ldots]$ tu parles si j'étais content ...

Exemple 19. Informations grammaticales sur tu parles dans le SFC

À côté des paramètres grammaticaux, d'autres informations apparaissent comme les marques de registre (souvent « fam. » ou «pop.»), ainsi que des informations liées à la fréquence, qui ne sont bien sûr pas établies à partir d'un corpus de référence, comme dans l'exemple 20, tiré du DEL.

C’est pas vrai ! « ce n'est pas possible ! sans blague !». [...]. La fréquence de cette expression depuis 1968-1970 est devenue très élevée $[\ldots]$

Exemple 20. Fréquence d'emploi de c'est pas vrai dans le DEL

Enfin, on notera dans les dictionnaires phraséologiques la présence quasi systématique d'exemples, construits ou parfois des citations pour le SFC.

De manière générale, le traitement est donc plus complet dans les dictionnaires phraséologiques, en particulier pour le SFC, pour les formules expressives.

\section{Conclusion}

$\mathrm{Au}$ terme de cet examen, qui doit encore être étoffé par un plus grand nombre d'expressions et d'ouvrages de référence, on peut dire que les ouvrages lexicographiques recensent dans une large mesure les formules qui nous intéressent, même si les traitements proposés présentent pour la plupart d'entre eux peu de cohérence. Cela traduit la difficulté à appréhender ce type de phraséologismes, pour lequel on dispose encore de peu de modélisations détaillées.

Parmi les ouvrages examinés, deux dictionnaires se détachent. D'une part le TLFi, qui analyse finement les contextes d'emploi et les propriétés syntaxiques. Par ailleurs, l'ouvrage de Bidaud, les Structures figées de la conversation, présente contrairement aux autres ouvrages, une microstructure assez systématique, qui intègre indications d'emploi, gloses, paramètres grammaticaux et exemples.

La nature phraséologique des phrases préfabriquées qui nous intéressent apparaît donc bien relevée par de nombreux ouvrages lexicographiques, dont la consultation, à côté des corpus oraux et écrits, pourra être précieuse pour l'étude des phraséologismes pragmatiques.

\section{Références bibliographiques}

Bally, Ch. 1909. Traité de stylistique française. Paris : Klincksieck.

Bidaud, F. 2002. Structures figées de la conversation: analyse contrastive français-italien. Bern : Peter Lang. 
Blanco, X. 2015. Les pragmatèmes: définition, typologie et traitement lexicographique. Verbum, 4(4), p $17-25$.

Blanco, X. et Mejri, S. 2018. Les pragmatèmes. Paris : Champion..

Debaisieux, J.-M., Benzitoun, Ch., Deulofeu, J. 2016. Le projet ORFEO : Un corpus d'études pour le français contemporain, Corpus, 15, 5. http://journals.openedition.org/corpus/2936.

Dostie, G. 2019. Paramètres pour définir et classer les phrases préfabriquées : La vengeance est un plat qui se mange froid. Bon appétit! Cahiers de lexicologie, 114, p. 27-61.

Fónagy I. 1997. Figement et changement sémantique. dans M. Martins-Baltar (dir..), La locution entre langue et usages. Fontenay-Saint-Cloud : ENS Éditions, p. 131-164.

Hausmann, F. 1996. La syntagmatique dans le TLF informatisé. In D. Piotrowski (ed.) Autour de l'informatisation $d u$ TLF, Actes du Colloque International de Nancy (29-31 mai 1995). Paris : Didier, 51-77.

Kauffer, M. 2013.Le figement des " actes de langage stéréotypés » en français et en allemand », Pratiques: théories, pratique, pédagogie, 159-160, p. 42-54.

Kauffer, M. 2019. Les "actes de langage stéréotypés" : essai de synthèse critique. Cahiers de lexicologie, 114, p. 149-171.

Klein, J.-R., Lamiroy, B. 2011. Routines conversationnelles et figement, dans J.-CL Anscombre et S. Mejri (dir.), Le figement linguistique : la parole entravée. Paris : Honoré Champion, p. 195-214.

Krzyżanowska, A., Grossmann, F. 2019. Pragmatèmes en contraste : de la modélisation linguistique au codage lexicographique. Lublin Studies in Modern Languages and Literature, 42(4), 252.

Krzyżanowska, A., Grossmann, F. (à paraître, 2020). Les formules expressives de la conversation en français, polonais et italien. Analyse contrastive. Lublin : Presses Universitaires de l'Université Marie Curie-Sklodowska.

López Simó, M. 2016. Fórmulas de la conversación. Propuesta de definición y clasificación con vistas a su traducción español-francés, francés-español. Thèse de doctorat. Université d'Alicante.

Mel'čuk, I. 2013.Tout ce que nous voulions savoir sur les phrasèmes, mais ..., Cahiers de Lexicologie 102, p. $129-149$.

Sokolowska, A. 2019. L'analyse des phraséologismes pragmatiques de structure C'est pas + adjectif, Mémoire de master 2 sciences du langage, parcours Linguistique, Université Grenoble-Alpes.

Tutin, A. 2019. Phrases préfabriquées des interactions: quelques observations sur le corpus CLAPI. Cahiers de Lexicologie, 114, p. 63-91. 


\section{Gloses élaborées pour les expressions examinées dans le projet Pragmalex. cf. l'ouvrage de Krzyżanowska, A., Grossmann, F. (à paraître) pour une description plus complète}

La formule C'EST PAS CROYABLE est utilisée quand le locuteur veut exprimer une profonde surprise, mêlée parfois de réporbation ou d'admiration, face à un phénomène inattendu.

1. La formule C'EST PAS VRAI ! est utilisée quand le locuteur veut exprimer son étonnement ou son incrédulité, avec parfois des traces d'admiration ou d'indignation, en réaction à un fait ou un événement qui lui est rapporté.

2. La formule C'EST PAS VRAI ! (parfois précédée de MAIS) peut avoir une valeur plus spécifiquement négative; elle accompagne alors une situation difficile vécue par le locuteur, et lui permet d'exprimer son mécontentement, son découragement ou sa lassitude.

La formule C'EST PAS POSSIBLE! est utilisée quand le locuteur veut exprimer sa surprise, son étonnement ou sa colère (parfois avec une nuance d'ironie) devant une nouvelle, un événement inattendus.

La formule C'EST UNE HONTE ! est utilisée lorsque le locuteur exprime son indignation devant un fait qu'il juge injuste, révoltant, ou inacceptable.

La formule C'EST UNE PLAIE est utilisée lorsque le locuteur veut qualifier très négativement une chose ou une personne insupportable.

La formule ENCORE HEUREUX est utilisée (parfois ironiquement) lorsque le locuteur veut évaluer positivement un fait dans une situation qui est globalement négative.

La formule TU PARLES ! marque tantôt l'approbation ou l'accord du locuteur, tantôt au contraire la désapprobation et le désaccord du locuteur, en réaction aux propos de son interlocuteur.

${ }^{1}$ Comme dans Je touche du bois.

${ }^{2} \mathrm{D}$ 'autres formules du même type sont bien entendu possibles comme qu'est-ce qu'il vous fallait ? (remarque d'un relecteur).

${ }^{3}$ Comme dans cet extrait du Corpus du Français Parlé Parisien :

c'est des choses auxquelles on (n') accorde aucune importance quand on est jeune enfin à mon époque +

+ et qu'on retrouve plus tard ++ comment dirais-je en regrettant d'avoir perdu quelque chose quoi.

(Montreuil, Mo01).

${ }^{4}$ Le projet d'étendre le corpus pour la version finale n'a pas été envisageable du fait du confinement et de la difficulté à accéder à certains ouvrages lexicographiques.

${ }^{5}$ Voir aussi le mémoire de Sokolowska (2019).

${ }^{6} \mathrm{https}: / /$ www.projet-orfeo.fr/

${ }^{7} \mathrm{https}$ ://www.lerobert.com/dictionnaires/francais/langue/dictionnaire-le-grand-robert-de-la-langue-

francaise-abonnement-annuel-3133099010289.html\#group-ouvrage-presentation

${ }^{8} \mathrm{http}: / /$ atilf.atilf.fr/

${ }^{9} \mathrm{https} / / /$ fr.wiktionary.org/wiki/

1040 correspond ici à 10 expressions pour 4 dictionnaires.

${ }^{11}$ De manière générale, le Wiktionnaire intègre une large nomenclature d'expressions polylexicales par rapport aux dictionnaires de langue plus traditionnels. On notera en particulier une catégorie de locutions appelées « locutions-phrases » qui renvoient à des « Suite de mots, figée par l'usage, formant une phrase et dans laquelle la fonction de chaque mot pris isolément n'est pas analysable » (Définition de Wiktionnaire).

${ }^{12} \mathrm{Ce}$ qui ne nous paraît pas tout à fait justifié. C'est une honte renvoie à une désapprobation morale, ce qui n'est pas le cas de c'est la honte qui renvoie davantage au sentiment de honte comme synonyme de gêne. 